\title{
Cervical canal stenosis and adjacent segment degeneration after anterior cervical arthrodesis
}

\author{
Jing Tao Zhang ${ }^{1} \cdot$ Jun Ming Cao ${ }^{1} \cdot$ Fan Tao Meng ${ }^{1} \cdot$ Yong Shen ${ }^{1}$
}

Received: 30 November 2014/Revised: 16 April 2015/Accepted: 16 April 2015/Published online: 23 April 2015

(C) The Author(s) 2015. This article is published with open access at Springerlink.com

\begin{abstract}
Purpose Adjacent segment degeneration (ASD) is known to occur after anterior cervical arthrodesis. However, it is not known whether cervical canal stenosis enhances the risk of ASD. The purpose of this study was to explore whether congenital stenosis could be used as a predictor of ASD after anterior cervical decompression and fusion (ACDF).

Methods We enrolled 141 patients who had undergone ACDF for cervical myelopathy and/or radiculopathy, and had at least 6 years of follow-up. In standard radiographs of cervical spine in lateral view, bony congenital stenosis was evaluated and all patients were divided into two groups: stenosis $(n=63)$ and non-stenosis $(n=78)$. Radiographic ASD was assessed according to the criteria of Kellgren and Lawrence and correlated with symptomatic ASD. Clinical and radiological parameters were compared between the groups. The primary outcome was the rate of radiographic ASD after initial ACDF. The incidence of symptomatic ASD was assessed by Kaplan-Meier method. Results Radiographic ASD and symptomatic ASD developed in $46.8 \%$ and $18.4 \%$ of all patients, respectively. There was a significant association between congenital stenosis and radiographic ASD. The area under the receiver operating characteristic curve of preoperative anteroposterior (AP) diameter of cervical canal for predicting radiographic ASD was $0.756 .13 .0 \mathrm{~mm}$ was the cutoff value
\end{abstract}

J. T. Zhang and J. M. Cao contributed equally to this study.

Yong Shen

houheicun@126.com

1 Department of Spinal Surgery, The Third Hospital of Hebei Medical University, No. 139 Ziqiang Road, Shijiazhuang 050051, Hebei, People's Republic of China of preoperative AP diameter of cervical canal predicting radiographic ASD. Kaplan-Meier analysis predicted a disease-free survival rate of symptomatic ASD in $97.2 \%$ of patients at 5 years and $78.0 \%$ at 10 years after ACDF. There was no significant difference in survival rates of the adjacent segment between the two groups via log-rank analysis $(P=0.102)$.

Conclusion Congenital stenosis can increase the rate of radiographic ASD after initial ACDF. The cutoff value of $13.0 \mathrm{~mm}$ for preoperative AP diameter of cervical canal had the highest validity for predicting radiographic ASD.

Keywords Adjacent segment degeneration - Anterior fusion - Cervical spine $\cdot$ Disc degeneration $\cdot$ Stenosis

\section{Introduction}

Anterior decompression of the cervical cord and interbody fusion for cervical radiculopathy and/or myelopathy were first introduced by Robinson and Smith [1] and by Cloward $[2,3]$. The development of adjacent segment degeneration (ASD) following anterior cervical decompression and fusion (ACDF) is well reported in the literature [4-15]. Within the past few decades, both in vivo and in vitro biomechanical evidence suggest that arthrodesis may augment stress on the unfused adjacent levels [16-21]. Hilibrand et al. [7] reported an annualized incidence of $2.9 \%$ per year for developing symptomatic ASD after singlelevel ACDF and estimated that about $25.6 \%$ of patients would have symptomatic ASD within 10 years of their index surgery. More than two-thirds of patients developing symptomatic ASD had failure of conservative treatment and required additional operative procedures. However, no agreement exists as to the etiology of ASD [7, 8, 22-24]. In 
patients with congenital cervical canal stenosis, the occurrence of ASD may lead to the development of new myelopathy and/or radiculopathy. However, to the best of our knowledge, few reports have described the association between a narrow cervical spinal canal and ASD. In this study, we investigate the relationship between congenital cervical canal stenosis and pathological changes of adjacent segment after ACDF.

\section{Materials and methods}

\section{Patient population}

We retrospectively reviewed 141 patients who had undergone ACDF for cervical degenerative disease between $\mathrm{C} 3$ and $\mathrm{C} 7$ and had at least 6 years of follow-up. Exclusion criteria included posttraumatic cervical disc herniation, a history of cervical spine surgery such as concomitant posterior decompression and fusion, preoperative degeneration (grade 2, 3, and 4) of adjacent segments based on the criteria of Kellgren and Lawrence [25], and follow-up of less than 6 years (Fig. 1). All patients underwent ACDF surgery in our hospital.

The study population consisted of 48 females and 93 males. The average age at the time of surgery was 51.2 years, ranging from 34 to 70 years. The average follow-up period was 9.7 years, ranging from 6 to 18 years. Demographic data included age at operation, gender, symptoms at presentation (radiculopathy vs. myelopathy vs. myeloradiculopathy) and length of follow-up. Surgical data collected included the number of fusion levels and type of bone graft (autograft vs. allograft).

\section{Surgical technique}

Under general anesthesia, a standard Smith-Robinson method was used to expose the cervical spine. After a complete discectomy and osteophytectomy were carried out, the endplate cartilage was symmetrically removed with a high-speed drill and curette until bleeding occurred. In all cases, an adequate decompression of cervical cord and the origin of nerve root was obtained. After confirming good pulsation of the thecal sac, an appropriate tricortical autograft or corticocancellous allograft was shaped into lordosis and inserted into the intervertebral space. Finally, a plate-and-screw system was applied to achieve anterior cervical fixation. After surgery, a Philadelphia cervical orthosis was applied for 4 weeks and a soft cervical collar was recommended for an additional 2 weeks.

\section{Radiological analysis}

Pre- and postoperative radiographs were obtained to assess the development of ASD. Kellgren and Lawrence diagnosed radiographic degeneration based on the following criteria: (1) formation of osteophytes; (2) periarticular ossicles; (3) cartilage narrowing with subchondral bone sclerosis; (4) small pseudocystic areas with sclerotic walls; (5) altered bone ends shape. Radiographic degeneration was divided into five grades as follows: grade 0 indicates no degenerative changes; grade 1 indicates doubtful
Fig. 1 Flow diagram showing patients allocation in the current study

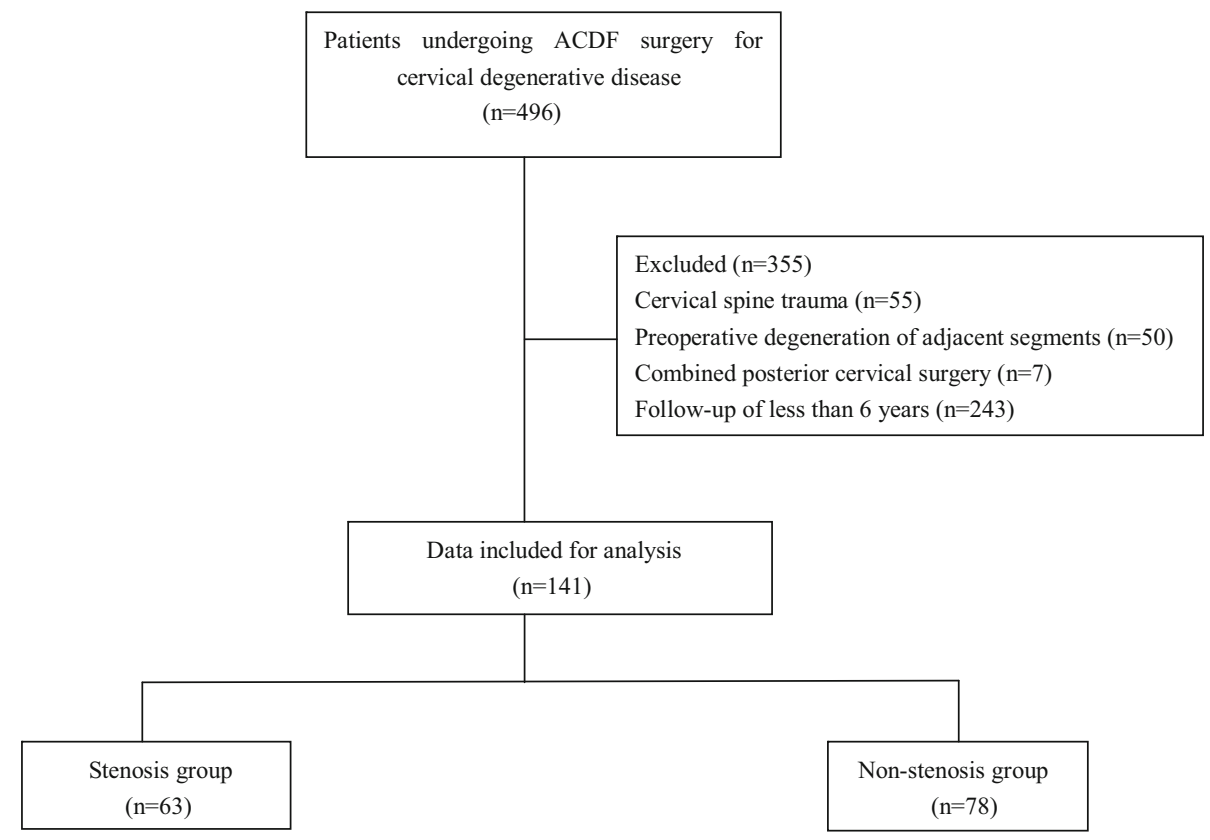


degeneration; grade 2 indicates minimal degeneration; grade 3 indicates moderate degeneration; and grade 4 indicates severe degeneration [25]. The sagittal canal diameter was measured on lateral neutral radiographs from the middle portion of the posterior surface of the vertebral body to the innermost cortical surface of the lamina. The anteroposterior (AP) diameter of the cervical canal was defined as the average of sagittal canal diameters from $\mathrm{C} 3$ to $\mathrm{C} 7$. Male patients whose sagittal diameter was $<14 \mathrm{~mm}$ and female patients whose sagittal diameter was $<13 \mathrm{~mm}$ at least at one level indicated the existence of developmental canal stenosis [26]. All measurements were undertaken using a picture archiving communication system (Siemens, Germany). All radiological data were reviewed by two blinded observers (J. T. Z and J. M. C) and the mean values were used. The diagnosis of symptomatic ASD was defined as a compressive lesion at the levels adjacent to the fusion segment, confirmed by magnetic resonance imaging (MRI) or myelography, and was considered as a new development of radiculopathy and/or myelopathy.

The primary outcome was the rate of radiographic ASD after initial ACDF. To evaluate whether congenital cervical canal stenosis could be a predictor of radiographic or symptomatic ASD, we divided patients into two groups: stenosis group and non-stenosis group. We compared the rate of radiographic and symptomatic ASD in stenosis and non-stenosis group using the Chi-square test. We evaluated the correlation between preoperative AP diameter of cervical canal and the radiographic ASD using logistic regression and Spearman's rho, which permit correlations between continuous variables and dichotomous variables to be made. Receiver operating characteristic (ROC) curves was conducted to evaluate the sensitivity and specificity of preoperative AP diameter of cervical canal for predicting radiographic ASD. The incidence of symptomatic ASD was analyzed using Kaplan-Meier survival analysis. To verify the reliability of the measured data, the intra-observer and inter-observer correlations were checked using a Kappa coefficient test. Data analysis was performed using SPSS software version 19.0 (SPSS Inc, Chicago, IL, USA).

\section{Results}

The Kappa coefficient test showed that the data used for this study were reliable $(0.82$ and 0.83 , respectively). For all patients, $56(39.7 \%)$ patients suffered from radiculopathy, $38(27.0 \%)$ patients from myelopathy and the remaining $47(33.3 \%)$ from myeloradiculopathy, preoperatively. The majority of patients underwent 1- or 2-level fusion (55 and 53, respectively), with 32 undergoing 3-level fusion, and 1 undergoing 4-level fusion. In 122
$(86.5 \%)$ autogenous bone graft was used and in 19 (13.5\%) allograft (Table 1).

The AP diameter of cervical spinal canal was $12.6 \pm 0.6 \mathrm{~mm}$ in stenosis group and $15.1 \pm 0.8 \mathrm{~mm}$ in non-stenosis group $(P=0.000)$. At last follow-up, the overall incidences of radiographic ASD and symptomatic ASD were $46.8 \%$ and $18.4 \%$, respectively. Although the occurrence of ASD was high after anterior cervical arthrodesis, overall clinical outcomes for patients were satisfied, with $85.1 \%$ of patients having good to excellent Robinson scores and $86.5 \%$ of patients having good to excellent Odom scores. Radiographic ASD occurred more frequently in stenosis group than in non-stenosis group: $61.9 \%$ vs. $34.6 \%$, respectively $(P=0.001$; Table 1 ; Fig. 2). However, the incidences of symptomatic ASD were not significantly different $(23.8 \%$ vs. $14.1 \%$, respectively; $P=0.139$; Table 1 ). Increase of preoperative AP diameter of cervical canal correlated with $(r=-0.442$; $P<0.001)$ radiographic adjacent segment degeneration (Fig. 3). The logistic regression for preoperative AP diameter of cervical canal and radiographic ASD found an OR of 0.457 (95\% confidence interval 0.335-0.624; $P<0.001$; Fig. 4). The area under the ROC curve of preoperative AP diameter of cervical canal for predicting radiographic ASD was 0.756 (95\% confidence interval $0.677-0.835 ; P<0.001)$. The cutoff value of $13.0 \mathrm{~mm}$ for preoperative AP diameter of cervical canal had the highest validity for predicting radiographic ASD. Kaplan-Meier survivorship analysis showed that predicted survivorship of the adjacent segments was $97.2 \%$ at postoperative 5 years and $78.0 \%$ at 10 years (Fig. 5). There was no significant difference in survival rates of the adjacent segment between the stenosis and non-stenosis groups via log-rank analysis $(P=0.102$; Fig. 6$)$. Of the 26 patients with symptomatic ASD after the initial ACDF, 15 patients had conservative treatment and new symptoms were relieved. Eleven patients required additional surgery after the initial ACDF because of recurrent myelopathy and/or radiculopathy caused by adjacent segment pathology. At the second surgery, an anterior fusion was performed in 5 patients, and 5 underwent laminoplasty. One patient disapproved second surgery because of old age.

\section{Discussion}

Anterior cervical decompression and fusion is a commonly applied procedure for cervical degenerative disease. However, arthrodesis of the cervical spine segments results in alteration to the physiological biomechanics of the spine $[9,15,27,28]$. Baba et al. [6] observed that $25 \%$ of patients undergoing anterior cervical fusion subsequently developed new spinal canal stenosis above fused segments 
Table 1 The difference of clinical and radiological parameters between stenosis and non-stenosis groups

\begin{tabular}{llll}
\hline Data & Stenosis $(n=63)$ & Non-stenosis $(n=78)$ & $P$ value \\
\hline Age at operation (years) & $51.6 \pm 5.4$ & $50.9 \pm 7.2$ & 0.555 \\
Sex (male:female) & $45: 18$ & $48: 30$ & 0.218 \\
AP diameter of cervical canal (mm) & $12.6 \pm 0.6$ & $15.1 \pm 0.8$ & 0.000 \\
Symptoms at presentation & & & 0.508 \\
Radiculopathy & 23 & 33 & \\
Myelopathy & 20 & 18 & 0.808 \\
Myeloradiculopathy & 20 & 27 & \\
Bone graft & & & \\
Autograft & 55 & 67 & 0.757 \\
Allograft & 8 & 11 & \\
The number of levels fused & & & \\
1 level & 23 & 32 & 0.304 \\
2 levels & 25 & 28 & 0.001 \\
3 levels & 15 & 17 & 0.139 \\
4 levels & 0 & 1 & \\
Follow-up period (years) & $10.0 \pm 2.8$ & $27(34.6 \%)$ & $11(14.1 \%)$ \\
Radiographic ASD & $39(61.9 \%)$ & & \\
Symptomatic ASD & $15(23.8 \%)$ & $9.5 \pm 2.5$ & \\
\hline AP and & & &
\end{tabular}

$A P$ anteroposterior, $A S D$ adjacent segment degeneration

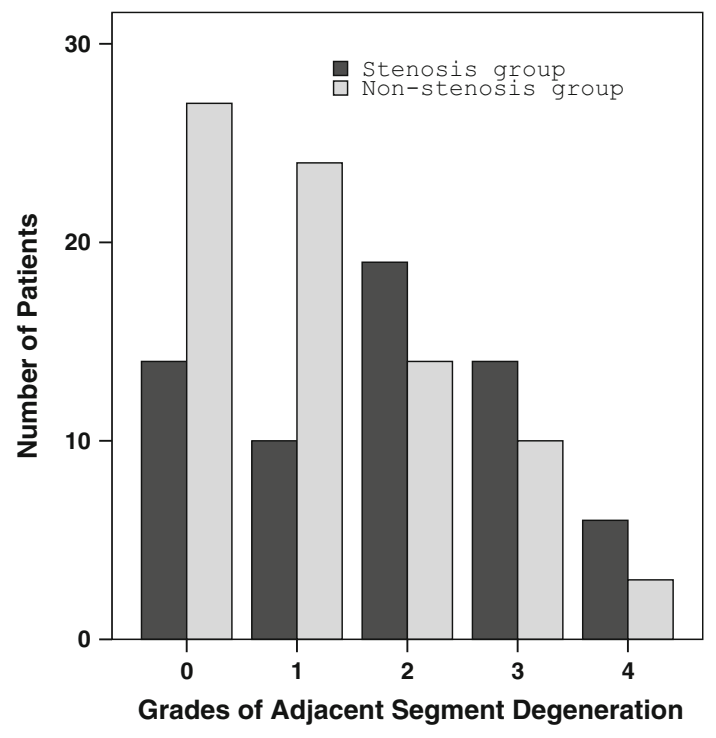

Fig. 2 A graph shows the number of patients in stenosis group and non-stenosis group sharing grades $0-4$ of radiographic adjacent segment degeneration according to the Kellgren-Lawrence classification

with an average of 8.5 years of follow-up. Bohlman et al. [29] reviewed 122 patients after anterior discectomy and fusion with an average of 6 years of follow-up and observed that $9 \%$ of all patients developed adjacent segment disease requiring additional surgery. Sufficient evidence is available to prove the development of adjacent segment changes. Nonetheless, many clinical studies did not support

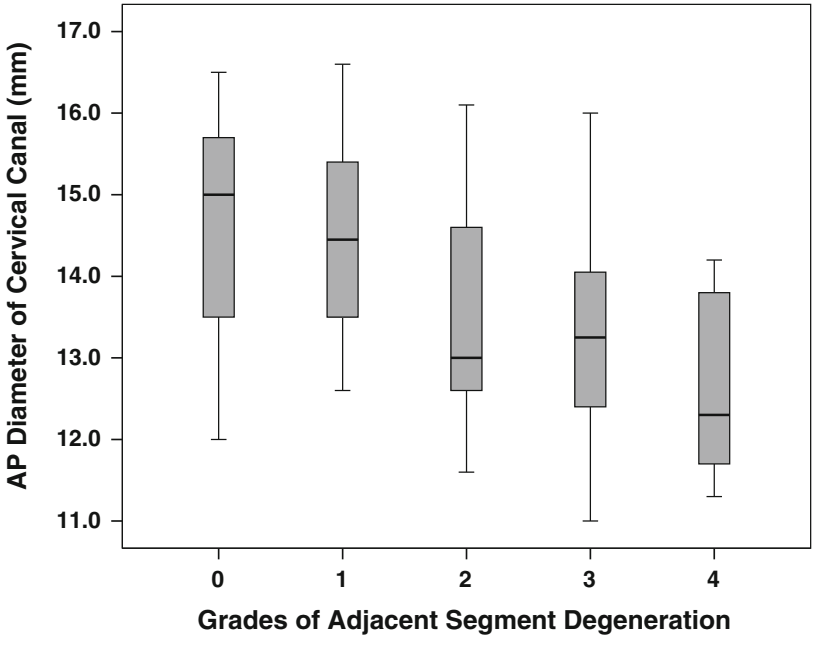

Fig. 3 A graph shows the correlation between the anteroposterior $(A P)$ diameter of cervical canal and the grade of radiographic adjacent segment degeneration. Box distribution, vertical bars SD, horizontal bar arithmetic average

this hypothesis. There are data available for comparison of fusion and nonfusion procedures to determine whether fusion itself might be causative in the development of ASD. Lunsford et al. [30] reported that the incidence of adjacent segment disease was not significantly different between patients undergoing $\mathrm{ACDF}$ and patients having discectomy only. In a prospective cohort study, Maldonado et al. [15] found that preservation of motion in patients undergoing cervical disc arthroplasty was not associated 


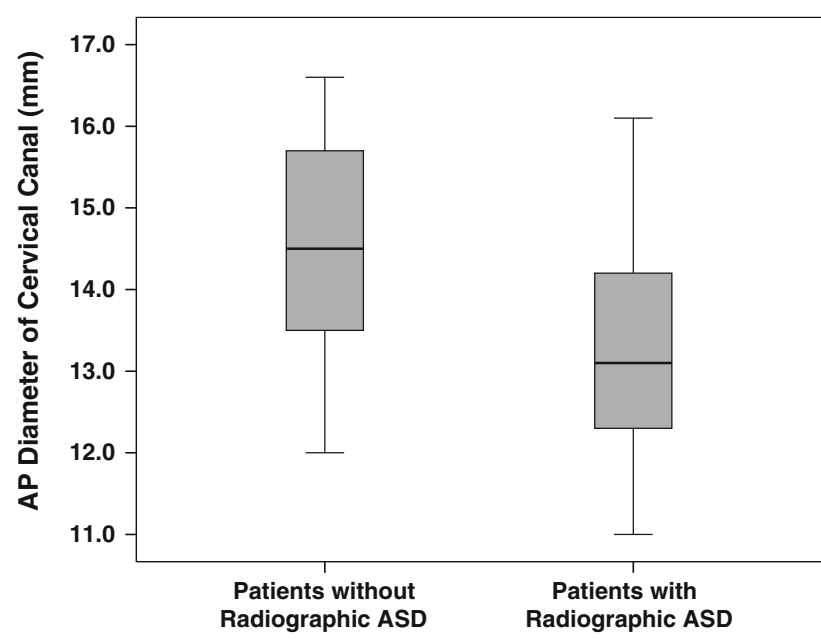

Fig. 4 A graph shows anteroposterior $(A P)$ diameter of cervical canal in patients without radiographic adjacent segment degeneration $(A S D)$ and in patients with radiographic ASD. Box distribution, vertical bars $\mathrm{SD}$, horizontal bar arithmetic average

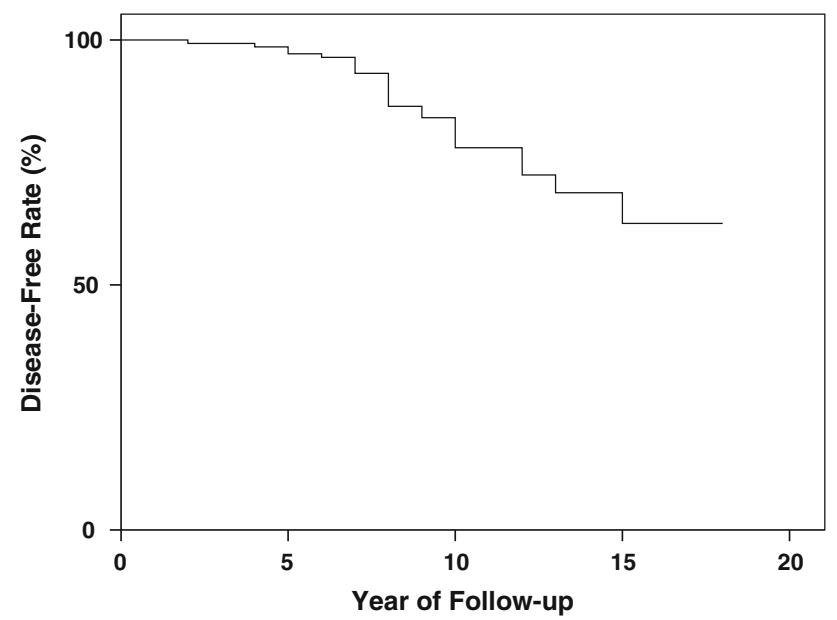

Fig. 5 Kaplan-Meier survival analysis of all patients undergoing anterior cervical decompression and fusion developing symptomatic adjacent segment degeneration over time

with a reduction of the rate of symptomatic ASD. Similarly, a few clinical studies suggest that ASD is the result of a natural progression of degenerative process and is not caused by the cervical spinal fusion itself.

The factors enhancing adjacent segment degenerative changes are still unclear. In neither of previous studies, however, was there any correlation between ASD and preoperative AP diameter of cervical canal. To better understand the development of ASD after anterior cervical arthrodesis and the overall prevalence of this problem, we focused our attention on the size of the cervical spinal canal and determined whether preoperative congenital stenosis predicted ASD at a minimum follow-up of 6 years. Morishita et al. [31] found that a congenitally narrow canal

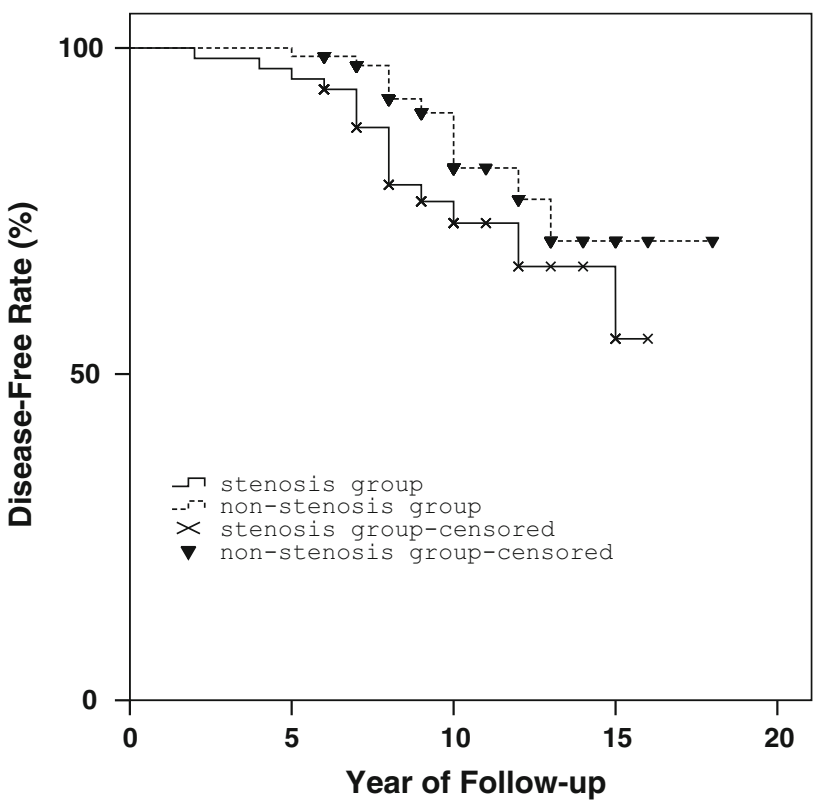

Fig. 6 Kaplan-Meier survival analysis for symptomatic adjacent segment degeneration, comparing stenosis group vs. non-stenosis group. There was no statistical difference between the two groups $(P=0.102)$

had different effects on cervical kinematics. In other words, subjects with a congenitally narrow cervical canal may be exposed to a large mechanical loading at the cervical spine. Their results suggest that cervical spinal canal diameter of less than $13 \mathrm{~mm}$ may be a risk factor for degenerative disc disease as measured in MRI studies. Therefore, we hypothesize that congenital stenosis would lead to an increased incidence of ASD following ACDF. In our study, we observed congenital cervical canal stenosis increased the rate of radiographic $\operatorname{ASD}(P=0.001)$. However, symptomatic ASD did not correlate with congenital stenosis in the current study $(P=0.139)$. Based on previous literatures $[7,22,29]$, there was a low incidence of symptomatic ASD after anterior cervical fusion. Because of the relatively small sample size in the current study, the statistical power of analysis on symptomatic ASD was low. Therefore, the result of symptomatic ASD between the two groups should therefore be regarded with caution. However, our result was similar to previous study, which showed that congenital stenosis does not appear to predict symptomatic ASD [32]. We found an OR of 0.457 between preoperative AP diameter of cervical canal and radiographic ASD, suggesting a narrow cervical spinal canal can be considered a risk factor for the development of radiographic ASD. Specifically, the odds of radiographic ASD decreased approximately by $50 \%$ for every 1 -mm increase in preoperative AP diameter of cervical canal. Therefore, we observed a narrow AP diameter of cervical canal was correlated with a higher rate of radiographic ASD and 
$13.0 \mathrm{~mm}$ was the cutoff value of preoperative AP diameter of cervical canal predicting radiographic ASD. We also compared the rate of symptomatic ASD development over time as a function of the size of cervical spinal canal via Kaplan-Meier analysis, but found no statistical significance between stenosis and non-stenosis groups. We speculated that the reason for this would be that patients with congenital cervical canal stenosis may have congenitally narrow cervical cords, and therefore, the conditions of the spinal cords were not more affected by the degenerative changes at the adjacent levels.

Regarding the treatment of symptomatic ASD, Hilibrand et al. [7] reported that only $28 \%$ of patients with symptomatic ASD had successful conservative treatment of the new symptoms. However, from our study, $57.7 \%$ of patients responded to careful conservative treatment, such as wearing a soft cervical collar, anti-inflammatory medications, and physiotherapy. When conservative treatment failed, ACDF or laminoplasty could be performed to resolve the symptoms of adjacent segment disease.

The present investigation may have several limitations. First, it is a retrospective observational study; therefore, other potential factors may affect the development of ASD. In addition, we do not have enough cases of symptomatic ASD after initial ACDF, and the statistical power of analysis on symptomatic ASD was low. Therefore, our result of symptomatic ASD should be interpreted with caution. Third, using AP diameter to determine the cervical canal stenosis is not the most precise technique. Finally, a large sample multi-center study is needed to gather extensive radiological and clinical data regarding ASD following ACDF.

\section{Conclusions}

In conclusion, patients who underwent anterior cervical arthrodesis for degenerative disease had a high incidence of radiographic ASD. The development of radiographic ASD was related to preoperative AP diameter of cervical spinal canal. The cutoff value of $13.0 \mathrm{~mm}$ for preoperative AP diameter of cervical canal had the highest validity for predicting radiographic ASD. Congenital cervical canal stenosis did not appear to contribute to symptomatic ASD. Because of the rarity of symptomatic ASD, the study may be underpowered. Further investigation may help to explain the mechanism by which such a correlation occurs. Surgeons should not be worried too much of symptomatic ASD when performing anterior cervical arthrodesis procedures for cervical degenerative disease even if preoperative congenital stenosis is present.

Acknowledgments The authors thank Ling De Kong, MD, and Jie $\mathrm{Li}, \mathrm{MD}$, for their contributions to this study.
Conflict of interest The authors declare that they have no conflicts of interest concerning this article.

Open Access This article is distributed under the terms of the Creative Commons Attribution 4.0 International License (http://creativecommons.org/licenses/by/4.0/), which permits unrestricted use, distribution, and reproduction in any medium, provided you give appropriate credit to the original author(s) and the source, provide a link to the Creative Commons license, and indicate if changes were made.

\section{References}

1. Robinson RA, Smith G (1955) Anterolateral cervical disc removal and interbody fusion for cervical disc syndrome. Bull Johns Hopkins Hosp 96:223-224

2. Cloward RB (1958) The anterior approach for removal of ruptured cervical disks. J Neurosurg 15:602-617

3. Cloward RB (1971) Complications of anterior cervical disc operation and their treatment. Surgery 69:175-182

4. Lundine KM, Davis G, Rogers M et al (2014) Prevalence of adjacent segment disc degeneration in patients undergoing anterior cervical discectomy and fusion based on pre-operative MRI findings. J Clin Neurosci 21:82-85

5. Bydon M, Xu R, Macki M et al (2014) Adjacent segment disease after anterior cervical discectomy and fusion in a large series. Neurosurgery 74:139-146

6. Baba H, Furusawa N, Imura $S$ et al (1993) Late radiographic findings after anterior cervical fusion for spondylotic myeloradiculopathy. Spine (Phila Pa 1976) 18:2167-2173

7. Hilibrand AS, Carlson GD, Palumbo MA et al (1999) Radiculopathy and myelopathy at segments adjacent to the site of a previous anterior cervical arthrodesis. J Bone Joint Surg Am 81:519-528

8. Ishihara H, Kanamori M, Kawaguchi Y et al (2004) Adjacent segment disease after anterior cervical interbody fusion. Spine $\mathbf{J}$ 4:624-628

9. Matsumoto M, Okada E, Ichihara D et al (2010) Anterior cervical decompression and fusion accelerates adjacent segment degeneration: comparison with asymptomatic volunteers in a ten-year magnetic resonance imaging follow-up study. Spine (Phila Pa 1976) 35:36-43

10. Coric D, Nunley PD, Guyer RD et al (2011) Prospective, randomized, multicenter study of cervical arthroplasty: 269 patients from the Kineflex|C artificial disc investigational device exemption study with a minimum 2-year follow-up: clinical article. J Neurosurg Spine 15:348-358

11. Hunter LY, Braunstein EM, Bailey RW (1980) Radiographic changes following anterior cervical fusion. Spine (Phila Pa 1976) 5:399-401

12. Yue WM, Brodner W, Highland TR (2005) Long-term results after anterior cervical discectomy and fusion with allograft and plating: a 5- to 11-year radiologic and clinical follow-up study. Spine (Phila Pa 1976) 30:2138-2144

13. Jawahar A, Nunley P (2012) Total disc arthroplasty and anterior cervical discectomy and fusion in cervical spine: competitive or complimentary? Review of the literature. Global Spine J 2:183-186

14. Robertson JT, Papadopoulos SM, Traynelis VC (2005) Assessment of adjacent-segment disease in patients treated with cervical fusion or arthroplasty: a prospective 2-year study. J Neurosurg Spine 3:417-423

15. Maldonado CV, Paz RD, Martin CB (2011) Adjacent-level degeneration after cervical disc arthroplasty versus fusion. Eur Spine J 20:403-407 
16. Eck JC, Humphreys SC, Lim TH et al (2002) Biomechanical study on the effect of cervical spine fusion on adjacent-level intradiscal pressure and segmental motion. Spine (Phila Pa 1976) 27:2431-2434

17. DiAngelo DJ, Foley KT, Morrow BR et al (2004) In vitro biomechanics of cervical disc arthroplasty with the ProDisc-C total disc implant. Neurosurg Focus 17:E7

18. Park DH, Ramakrishnan P, Cho TH et al (2007) Effect of lower two-level anterior cervical fusion on the superior adjacent level. J Neurosurg Spine 7:336-340

19. Puttlitz CM, Rousseau MA, Xu Z et al (2004) Intervertebral disc replacement maintains cervical spine kinetics. Spine (Phila Pa 1976) 29:2809-2814

20. Kretzer RM, Hsu W, Hu N et al (2012) Adjacent-level range of motion and intradiscal pressure after posterior cervical decompression and fixation: an in vitro human cadaveric model. Spine (Phila Pa 1976) 37:E778-E785

21. Shin DA, Yi S, Yoon DH et al (2009) Artificial disc replacement combined with fusion versus two-level fusion in cervical twolevel disc disease. Spine (Phila Pa 1976) 34:1153-1159 discussion 1160-1161

22. Hilibrand AS, Robbins M (2004) Adjacent segment degeneration and adjacent segment disease: the consequences of spinal fusion? Spine J 4:190S-194S

23. Kulkarni V, Rajshekhar V, Raghuram L (2004) Accelerated spondylotic changes adjacent to the fused segment following central cervical corpectomy: magnetic resonance imaging study evidence. J Neurosurg 100:2-6

24. Kawakami M, Tamaki T, Yoshida M et al (1999) Axial symptoms and cervical alignments after cervical anterior spinal fusion for patients with cervical myelopathy. J Spinal Disord 12:50-56
25. Kellgren JH, Lawrence JS (1957) Radiological assessment of osteoarthrosis. Ann Rheum Dis 16:494-502

26. Higo M, Sakou T, Suzuki Y et al (1984) Roentgenological study of the antero-posterior diameter in cervical developmental canal stenosis. Rinsho Seikei Geka 19:361-366

27. Murrey D, Janssen M, Delamarter R et al (2009) Results of the prospective, randomized, controlled multicenter Food and Drug Administration investigational device exemption study of the ProDisc-C total disc replacement versus anterior discectomy and fusion for the treatment of 1-level symptomatic cervical disc disease. Spine J 9:275-286

28. Mummaneni PV, Burkus JK, Haid RW et al (2007) Clinical and radiographic analysis of cervical disc arthroplasty compared with allograft fusion: a randomized controlled clinical trial. J Neurosurg Spine 6:198-209

29. Bohlman HH, Emery SE, Goodfellow DB et al (1993) Robinson anterior cervical discectomy and arthrodesis for cervical radiculopathy. Long-term follow-up of one hundred and twenty-two patients. J Bone Joint Surg Am 75:1298-1307

30. Lunsford LD, Bissonette DJ, Jannetta PJ et al (1980) Anterior surgery for cervical disc disease. Part 1: treatment of lateral cervical disc herniation in 253 cases. J Neurosurg 53:1-11

31. Morishita Y, Naito M, Hymanson H et al (2009) The relationship between the cervical spinal canal diameter and the pathological changes in the cervical spine. Eur Spine J 18:877-883

32. Eubanks JD, Belding J, Schnaser E et al (2013) Congenital stenosis and adjacent segment disease in the cervical spine. Orthopedics 36:e1251-e1255 